Kristine Hillenkötter*, Maria Huber, Dr. Ursula Stanek und Gerald Steilen

\title{
Das Kompetenzzentrum für die Lizenzierung elektronischer Ressourcen im DFG-geförderten System der „Fachinformationsdienste für die Wissenschaft" (FID): Betriebsorganisation, Verhandlung und Bereitstellung von FID-Lizenzen - ein Statusbericht
}

DOI 10.1515/bfp-2016-0014

Zusammenfassung: Seit 2014 fördert die Deutsche Forschungsgemeinschaft (DFG) den Aufbau eines Kompetenzzentrums für die Lizenzierung elektronischer Ressourcen im DFG-geförderten System der „Fachinformationsdienste für die Wissenschaft" (FID). Am Aufbau beteiligt sind die Niedersächsische Staats- und Universitätsbibliothek in Göttingen (SUB Göttingen), die Staatsbibliothek zu Berlin (SBB) und die Verbundzentrale des Gemeinsamen Bibliotheksverbundes (VZG). Der Betrieb des Kompetenzzentrums erfolgt parallel an den Standorten Göttingen und Berlin. Die zunächst für den Zeitraum 2014-2016 terminierten Projektaktivitäten werden von der SUB Göttingen koordiniert.

Das Kompetenzzentrum für Lizenzierung agiert bundesweit als Serviceprovider, um die Fachinformationsdienste (FID) bei der Wahrnehmung ihrer Aufgaben zu unterstützen. Neben der Verhandlung, Lizenzierung und überregionalen Bereitstellung digitaler Medien bietet es die Entwicklung geeigneter Lizenz- und Geschäftsmodelle sowie Dienstleistungen zum Management der mit den Lizenzen erworbenen Daten und zum Aufbau entsprechender Mehrwertdienste an. Die Angebote des Kompetenzzentrums werden bedarfsorientiert und im direkten Kontakt mit den Fachinformationsdiensten weiterentwickelt.

Der vorliegende Beitrag gibt Auskunft über den aktuellen Stand der Betriebsorganisation sowie der Verhandlung und Bereitstellung von FID-Lizenzen zur Mitte der Projektlaufzeit.

\footnotetext{
*Kontaktperson: Kristine Hillenkötter, hillenkoetter@sub.uni-goettingen.de

Maria Huber, maria.huber@sub.uni-goettingen.de Dr.Ursula Stanek, ursula.stanek@sbb.spk-berlin.de Gerald Steilen, steilen@gbv.de
}

Schlüsselwörter: Überregionale Lizenzierung; Kompetenzzentrum für Lizenzierung; Fachinformationsdienste für die Wissenschaft (FID)

The Center of Competence for Licensing Electronic Resources within the Framework of the "Specialised Information Services for Research " (Fachinformationsdienste für die Wissenschaft, FID), funded by the DFG: Business Organisation, Negotiation and Allocation, a Status Report

Abstract: Commencing in 2014, the Deutsche Forschungsgemeinschaft (DFG) has been promoting the development of a Centre of Competence for Licensing Electronic Resources in the context of the "Specialised Information Services for Research" (FID). Participants of the installation of the Centre are the Niedersächsische Staats- und Universitätsbibliothek in Göttingen (SUB Göttingen), the Staatsbibliothek zu Berlin (SBB) and the Verbundzentrale des Gemeinsamen Bibliotheksverbundes (VZG = mutual library union). The teams at the libraries in Göttingen and Berlin work simultaneously. For the first period of the project, from 2014 through 2016, the activities of both teams were coordinated by the SUB Göttingen. The Centre of Competence for Licensing operates nationwide as a service provider to support the work of the Specialised Information Services for Research (FID). Additionally to negotiating, licensing and allocating of digital media nationwide it offers development of suitable license models and business models as well as management services concerning the value added services for the data delivered with the licenses. Offers were designed and submitted on demand, always in direct communication with the Specialised Information Services for Research. The following article will inform about the business organization, negotiations, and allocations so fare at half-time of the project. 
Keywords: Nationwide licensing; Centre of Competence for Licensing; Specialised Information Services for Research (FID)

Inhalt

1 Hintergrund ..................

2 Aufbau des Kompetenzzentrums für Lizenzierung ................ 34

2.1 Selbstverständnis als Dienstleister für die FID . . 35

2.2 Service- und Beratungsangebote . . . . . . . . 35

2.3 Betriebsorganisation ............. 36

3 FID-Lizenzen .................. 38

4 Verhandlung von FID-Lizenzen . . . . . . . . . . 39

4.1 Nutzerkreismodelle der FID . . . . . . . . . . . . . 39

4.2 Lizenz- und Geschäftsmodelle für FID-Lizenzen . 39

4.3 Interdependenzen zwischen FID-Lizenzen und bestehenden Beschaffungsstrukturen ...... 40

4.4 Umsetzbarkeit der Förderkriterien . . . . . . . 41

4.5 Werkstattbericht: Bearbeitung von Verhandlungsaufträgen der FID . . . . . . . . . . . .

5 Bereitstellung von FID-Lizenzen . . . . . . . . . 44

5.1 Bereitstellungsplattform des Kompetenzzentrums (KfL-ERMS) . . . . . . . . . . . . . 44

5.2 Registrierung und Authentifizierung . . . . . . . 46

5.3 Bereitstellungsmodelle und Kommunikations-

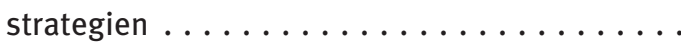

5.4 Erschließung von FID-Lizenzen in den Nachweis-

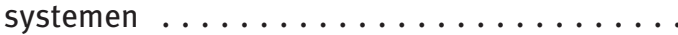
48

\section{(a)}

$$
\text { gen }
$$

\section{gen} verbundes (VZG). Der Betrieb des Kompetenzzentrum(s) für Lizenzierung (KfL) erfolgt parallel an den Standorten Göttingen und Berlin. Die Aktivitäten werden von der SUB Göttingen koordiniert.

\section{Aufbau des Kompetenzzentrums für Lizenzierung ${ }^{3}$} handlung, Lizenzierung und der überregionalen Bereit-

\section{Hintergrund}

Seit 2014 fördert die Deutsche Forschungsgemeinschaft $(\mathrm{DFG})^{1}$ den Aufbau eines Systems der „Fachinformationsdienste für die Wissenschaft“ (FID), das in Ergänzung zu den lokalen Informationsinfrastrukturen der Hochschulen und Forschungseinrichtungen der Versorgung von Wissenschaftlern aller Fachrichtungen in Deutschland mit digitalen Medien und Informationsangeboten für den wissenschaftlichen Spitzenbedarf dient. ${ }^{2}$ Es löst die seit 1949 bestehende Förderung der "Sondersammelgebiete“ ab. Um die einzelnen Fachinformationsdienste zugunsten ihrer fachlichen Arbeit zu entlasten, wurde systemimmanent eine Querschnittsaufgabe für die Beschaffung und Bereit-

1 www.dfg.de (letzter Zugriff 15.11.2015 bei allen Internetquellen). 2 Vgl. DFG-Förderprogramm „Fachinformationsdienste für die Wissenschaft“: http://www.dfg.de/foerderung/programme/infrastruktu $\mathrm{r} /$ lis/lis_foerderangebote/fachinformationsdienste_wissenschaft. und die Verbundzentrale des Gemeinsamen Bibliotheks-

Die Aufgabe des Kompetenzzentrums besteht in der Verstellung kostenpflichtiger elektronischer Ressourcen für die FID. Es soll diese sog. „FID-Lizenzen“ in der Praxis etablieren und dafür geeignete Lizenz-, Geschäfts- und

stellung kostenpflichtiger elektronischer Ressourcen definiert, die besondere fachliche Expertisen sowie infrastrukturelle Voraussetzungen erfordert.

In diesem Rahmen unterstützt die DFG auch den Aufbau eines Kompetenzzentrums für die Lizenzierung elektronischer Ressourcen im Kontext der DFG-geförderten „Fachinformationsdienste für die Wissenschaft“ (FID), das diese Querschnittsaufgabe wahrnehmen soll. Es handelt sich hierbei um eine zunächst auf drei Jahre angelegte Projektaktivität (2014-2016). Projektpartner sind die Nieersächsische Staats- und Universitätsbibliothek in GöttinBereitstellungsmodelle, Workflows und die organisatorischen Voraussetzungen entwickeln. ${ }^{4}$

Damit werden parallele Handlungsfelder berührt, für die auf überregionaler Ebene bereits Strukturen und Entwicklungen existieren. Hier sind zum einen die Aktivitäten im Kontext der etablierten Beschaffungsstrukturen für die überregionale Lizenzierung, Verwaltung und Bereitstellung elektronischer Medien zu nennen (National- und Allianz-Lizenzen, Konsortien). ${ }^{5}$ Zum anderen werden hier die Bemühungen um die Entwicklung eines Querschnitts-

3 Vgl. Hillenkötter, Kristine: Aufbau des „Kompetenzzentrums für die Lizenzierung elektronischer Ressourcen“ im Kontext der DFGgeförderten „Fachinformationsdienste für die Wissenschaft“. Bibliothekartag 2014. Vortragsfolien: https://opus4.kobv.de/opus4-bib-inf o/frontdoor/index/index/docId/1455.

4 Vgl. Deutsche Forschungsgemeinschaft: „Richtlinien Fachinformationsdienste für die Wissenschaft“. 2015, S.11f.: http://www.dfg.de/f ormulare/12_102/12_102_de.pdf.

5 Vgl. hier vor allem die Zusammenführung der Aktivitäten zu den Konsortien und Allianz-Lizenzen in der GASCO (German, Austrian, and Swiss Consortia Organisation) unter: https://www.hbz-nrw.de/a ngebote/digitale_inhalte/gasco/ sowie die Bereitstellungsplattform für die Nationallizenzen unter: www.nationallizenzen.de. 
bereiches „Hosting und Langzeitarchivierung“ im FID-System, die Entwicklung einer nationalen Hosting-Strategie und -Lösung sowie die Bereitstellung von Lösungen für die Langzeitarchivierung elektronischer Ressourcen auf nationaler Ebene tangiert. ${ }^{6}$ Diesen Umstand galt es bei der Planung des Kompetenzzentrums zu berücksichtigen.

Zudem steht der Aufbau des Kompetenzzentrums in enger Relation zur Entwicklung der FID, für die es tätig werden sollte ${ }^{7}$ : Bei Projektbeginn zu Anfang des Jahres 2014 wurden bereits erste geisteswissenschaftliche FID eingerichtet, gleichzeitig bereiteten die naturwissenschaftlichen Disziplinen in der ersten Hälfte des Jahres 2014 ihre FID-Anträge vor. Beide Fachcluster stellten die ersten potentiellen Kunden des Kompetenzzentrums dar, das daher in der Lage sein musste, sehr schnell produktiv zu werden, um bereits Mitte 2014 erste belastbare Verhandlungsaufträge vorzulegen und 2015 die Freischaltung erster Ressourcen gewährleisten zu können.

Vor diesem Hintergrund wurde für den Aufbau des Kompetenzzentrums ein pragmatischer Ansatz gewählt, der nach Möglichkeit bereits vorhandene Erfahrungen und Entwicklungen nachnutzt. Er setzt auf die Adaption und Anpassung von bereits etablierten und erprobten Lizenzund Bereitstellungsmodellen, Infrastrukturen und Workflows aus den Kontexten der National- und Allianz-Lizenzen sowie der CrossAsia-Lizenzen für den FID-Kontext, ohne dabei die parallelen Handlungsfelder auf nationaler Ebene aus dem Blick zu verlieren.

\subsection{Selbstverständnis als Dienstleister für die FID}

Das Kompetenzzentrum agiert bundesweit als zentraler Serviceprovider für die FID, um diese bei der Wahrnehmung ihrer Aufgaben zu unterstützen.

Es übernimmt im Auftrag der FID die Verhandlung, Lizenzierung und überregionale Bereitstellung ausgewählter digitaler Medien und handelt dabei in der Regel

6 Vgl. hier vor allem das DFG-Projekt NatHosting (http://www.na thosting.de) sowie die seitens der DFG formulierte Perspektive zur Entwicklung eines zweiten Querschnittsbereiches im FID-System zum Thema „Hosting und Langzeitarchivierung“ in: Deutsche Forschungsgemeinschaft: „Richtlinien Fachinformationsdienste für die Wissenschaft“. 2015, S.13: http://www.dfg.de/formulare/12_102/12_102_ de.pdf.

7 Vgl. den Zeitplan zur Überführung der DFG-geförderten Sondersammelgebiete in DFG-geförderte Fachinformationsdienste für die Wissenschaft: http://www.dfg.de/foerderung/programme/infrastruk tur/lis/lis_foerderangebote/fachinformationsdienste_wissenschaft/u eberfuehrung_sondersammelgebiete. auf Nachfrage durch die FID (Auftragserteilung), aber auch proaktiv (Beratungsleistungen). Dabei vermittelt es stets zwischen den Interessen der FID und den Möglichkeiten der Verlage. Bei der Verhandlung von FID-Lizenzen sind die Rollen zwischen KfL und FID klar definiert: Als Dienstleister kümmert sich das KfL im Auftrag der FID zentral um die Verhandlung, die Vorbereitung der Lizenzverträge und die Bereitstellung der Lizenzen. Lizenznehmer selbst ist der FID, der die Produkte auswählt, den fachlich definierten Nutzerkreis festlegt, die Lizenzverträge unterzeichnet, die Lizenzkosten begleicht, seine Nutzer über die Lizenzangebote informiert und die Berechtigungen im Bereitstellungsprozess vergibt.

\subsection{Service- und Beratungsangebote}

Das Kompetenzzentrum bietet den FID ein umfangreiches Service- und Beratungsangebot für die Erwerbung und Nutzung von FID-Lizenzen an: Neben der Verhandlung, Lizenzierung und überregionalen Bereitstellung digitaler Medien gehören auch die Entwicklung geeigneter Lizenzund Geschäftsmodelle sowie Dienstleistungen zum Management der mit den Lizenzen erworbenen Daten und zum Aufbau entsprechender Mehrwertdienste zum Serviceportfolio ${ }^{8}$ :

Verhandlung und Erwerb von FID-Lizenzen

- Produktanalysen und Marktsondierung

- Beratung bei der Produktauswahl

- Beratung hinsichtlich der bedarfsgerechten Gestaltung von Titelpaketen für Zeitschriften und E-Books, ggf. auch bei modular angebotenen Datenbanken

- Beratung über Möglichkeiten und Erfolgsaussichten der Verhandlungen in Relation zu den Anforderungen der FID-Grundsätze

- Beratung zu geeigneten Lizenzmodellen

- Entwicklung von bedarfsorientierten Lizenz- und Geschäftsmodellen

- Lizenzverhandlungen

- Vertragsvorbereitung

- Unterstützung bei Rechnungsstellung und Fakturierung

8 Vgl. http://www.fid-lizenzen.de/ueber-fid-lizenzen/beratungs-un d-serviceangebote. Angebote für die Langzeitarchivierung der digitalen Objekte gehören nicht zum Angebotsspektrum des Kompetenzzentrums. Hier wird das Kompetenzzentrum für die FID nur beratend tätig werden und ggf. auf die Entwicklungen auf nationaler Ebene bzw. hinsichtlich der geplanten Etablierung eines systemimmanenten Querschnittsbereiches „Hosting und Langzeitarchivierung“ verweisen. 
- Beratung über Möglichkeiten und Grenzen der erworbenen Nutzungsrechte

- Ggf. Vermittlung der Bereitstellung über externe PPUPlattform(en)

\section{Überregionale Bereitstellung von FID-Lizenzen}

- Bereitstellung der Zugangsinformationen für Verlagsserver bzw. Eigenhosting/Freischaltung

- Betrieb einer eigenen Managementplattform für FIDLizenzen (ERMS)

- Bereitstellung von Schnittstellen zur Integration fachlicher Angebote sowie Anmelde- und Kontaktinformationen in die Webpräsenzen der FID

- Bereitstellung der technischen Infrastruktur zur Authentifizierung der Nutzer

Lizenzadministration und Nutzerkommunikation

- Zentrale Nutzerverwaltung

- Unterstützung alternativer, dezentraler Nutzerverwaltung durch den FID durch Bereitstellung einer Standard-Schnittstelle

- Nutzerbetreuung in Kooperation und Abstimmung mit dem FID

- „Helpdesk“ für lizenzbezogene Nutzerkommunikation

- Kommunikation mit den Anbietern in Abstimmung

- Gewährleistung des Rechtemanagements (Zugriffsrechte, Nutzungskonditionen, Zugangsinformationen $^{9}$ )

- $\quad$ Bedienung der Nachweisinstrumente ERMS, EZB, ZDB und DBIS

- EZB- und ZDB-Konsortialadministration für elektronische Zeitschriften

- Bereitstellung von Nutzungsstatistiken

\section{Datenmanagement}

- Beschaffung der mit den Lizenzen erworbenen Metadaten und digitalen Objekte von den Anbietern

- Dokumentation der Metadaten und digitalen Objekte über definierte Templates

- Beratung und Angebote zum Hosting von digitalen Objekten mit dem FID

- Lizenzverwaltung

- Beratung und Vermittlung von Angeboten für die Langzeitarchivierung von Metadaten und digitalen Objekten

- Speicherung der Metadaten und digitalen Objekte

- Konversion und Bereitstellung von Metadaten zur Nachnutzung durch Verbünde und Katalogsysteme

Mehrwertdienste auf Basis der erworbenen Daten und Nutzungsrechte

- Beratung über Möglichkeiten und Grenzen von Mehrwertdiensten auf Basis der erworbenen Daten und Nutzungsrechte

- Volltextindexierung und spezielle Indexierungsarbeiten

- Hilfestellungen für die Einrichtung von automatisierten Auswertungsdiensten (Text and Data Mining)

- Angebote zur Einbindung von Funktionalitäten wie OpenLinking, Schnittstellen, Personalisierungsfunktionen, interaktive und multimediale Elemente etc.

- Unterstützung bei der Einbindung der erworbenen Materialien in digitale Semesterapparate, virtuelle Forschungsbibliotheken, virtuelle Forschungsumgebungen etc.

- Ggf. weitere Hilfestellung für die Integration der Daten in das Servicespektrum des FID (z. B. im Rahmen eines Discovery Systems)

Die Angebote des Kompetenzzentrums werden bedarfsorientiert und im direkten Kontakt mit den Fachinformationsdiensten weiterentwickelt.

\subsection{Betriebsorganisation}

An der SUB Göttingen und der Staatsbibliothek zu Berlin wurde jeweils ein Serviceteam zur Bearbeitung der FIDAufträge aufgebaut. Die Serviceteams an beiden Standorten arbeiten parallel und werden von der SUB Göttingen koordiniert. Die Arbeitsteilung zwischen beiden Teams erfolgt nach interner Absprache, wobei die an beiden Standorten vorhandenen fachlichen und bibliothekarischen Spezialkenntnisse aus anderen Lizenzierungskontexten nachgenutzt werden. $\mathrm{Zu}$ jedem Team gehört jeweils eine „Hotline“ für die lizenzbezogene Nutzerkommunikation. Jeder FID-Kunde wird nach zentraler Kontaktaufnahme mit dem $\mathrm{KfL}^{10}$ einem der beiden Teams zugewiesen und

9 Mit Zugangsinformationen ist gemeint, auf welcher Plattform und mit welchen Verfahren - Freischaltung für welche IP-Bereiche, VPN, Shibboleth, (Rewriting) Proxy usw. - die Nutzer auf eine Ressource zugreifen können. Diese Informationen sind u.a. zum Betrieb von Rechercheplattformen (z. B. Discovery-Systeme) notwendig.
10 Die zentrale Kontaktaufnahme erfolgt über die Website des KfL unter: www.fid-lizenzen.de oder direkt über den Funktionsaccount info@fid-lizenzen.de. 


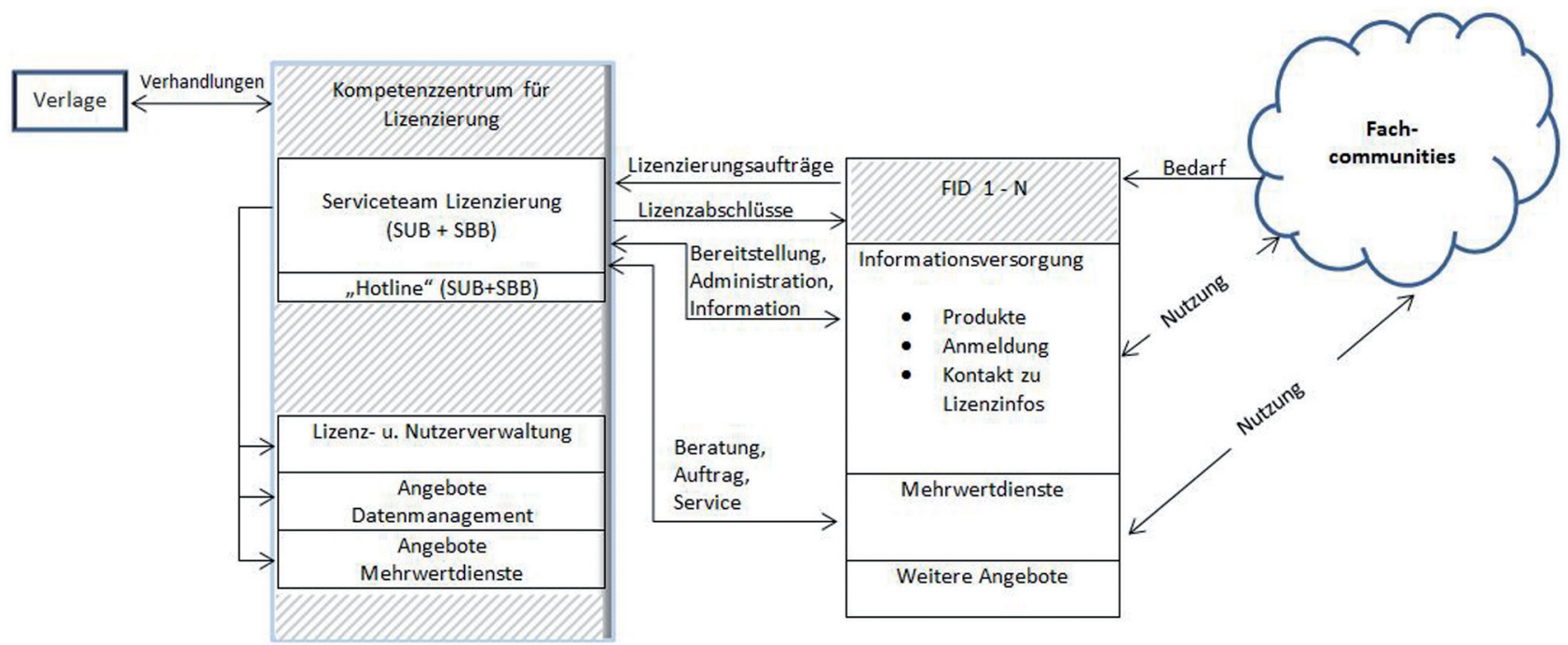

Abb. 1: Servicekonzept des Kompetenzzentrums für Lizenzierung (KfL)

dort mit allen Anliegen betreut (vgl. Abb.1). Er findet dort verlässliche und kontinuierlich verfügbare Ansprechpartner. Zur Dokumentation der Auftragserteilung und des verabredeten Leistungsumfangs schließt das Kompetenzzentrum mit den FID-Kunden sog. „Serviceverträge“ ${ }^{\text {“11 }}$, für den Abschluss von FID-Lizenzen setzt das Kompetenzzentrum einen Mustervertrag ein, der auf Basis der Musterlizenz für die Allianz-Lizenzen entwickelt wurde. ${ }^{12}$ Die Aufträge der FID werden nach definierten, flexibel handhabbaren Prozessabläufen in enger Abstimmung mit den FID abgewickelt. ${ }^{13}$

Die Verbundzentrale des Gemeinsamen Bibliotheksverbundes unterstützt den Servicebetrieb mit der dafür erforderlichen technischen Infrastruktur. Zentrales Element für die überregionale Bereitstellung und Nutzerverwaltung ist dabei das auf der Grundlage des bereits bewährten Content Management Systems für die Nationallizenzen ${ }^{14}$ im Rahmen des Projektes neu entwickelte KfL-ERMS, das für die Registrierung und Authentifizierung der Nutzer, die Gewährleistung des Rechtemanagements sowie die Nutzerund Produktverwaltung genutzt wird (vgl. Abb. 2). Hinzu treten die Infrastruktur für die Speicherung der mit den Lizenzen erworbenen Daten sowie Angebote zum Eigenhosting. Ergänzend bietet die Staatsbibliothek zu Berlin

11 Vgl. http://www.fid-lizenzen.de/ueber-fid-lizenzen/servicever trag-fid-kfl.

12 Vgl. http://www.fid-lizenzen.de/ueber-fid-lizenzen/dateien/fidmusterlizenz-dezember-2014.

$13 \mathrm{Vgl}$. http://www.fid-lizenzen.de/ueber-fid-lizenzen/kooperationzwischen-kompetenzzentrum-und-fid.

14 www.nationallizenzen.de. den FID die Nachnutzung ihrer Infrastruktur zum Eigenhosting von CD-ROM-Datenbanken an.

Außerhalb des Infrastrukturkonzeptes des Kompetenzzentrums stehen die von der Bayerischen Staatsbibliothek und den Zentralen Fachbibliotheken betriebenen und mit DFG-Förderung entwickelten Pay-per-Use-Plattformen für die überregionale Bereitstellung von elektronischen Medien zur Verfügung. Sofern die überregionale Bereitstellung lizenzierter Ressourcen aufgrund des gewählten Lizenzmodells über diese Plattformen notwendig ist, nimmt das Kompetenzzentrum mit den Betreibern der jeweils geeigneten Plattformen Kontakt auf und vermittelt für den jeweiligen FID die Bereitstellung der betreffenden Ressource über diese Plattform. Auf diese Weise wird ein seit Jahren für den Kontext der Sondersammelgebiete bestehendes Bereitstellungsangebot für den FID-Kontext nachgenutzt.

Entsprechend dem Selbstverständnis des KfL sind die Leistungen des Kompetenzzentrums für die FID primär dezentral über die Webpräsenzen der FID als deren Dienstleistungen für ihre Fachcommunities sichtbar, ein zentraler Einstieg über die Webpräsenz des KfL ist jedoch möglich.

Der Servicebetrieb des Kompetenzzentrums ist inzwischen voll entfaltet und hat sich in der Praxis bewährt. Letzter und wesentlicher Meilenstein in diesem Prozess war die Freigabe des neu entwickelten KfL-ERMS am 1. September 2015 für die Bereitstellung von FID-Lizenzen. Alle Kernaufgaben des KfL werden damit in den dafür vorgesehenen Strukturen abgewickelt. Perspektivisch gilt es jetzt noch, die unter den Oberbegriffen „Datenmanagement“ und „Mehrwertdienste auf Basis der erworbenen Daten und Nutzungsrechte“ definierten Serviceangebote des KfL im Dialog mit den FID weiter auszubauen. 


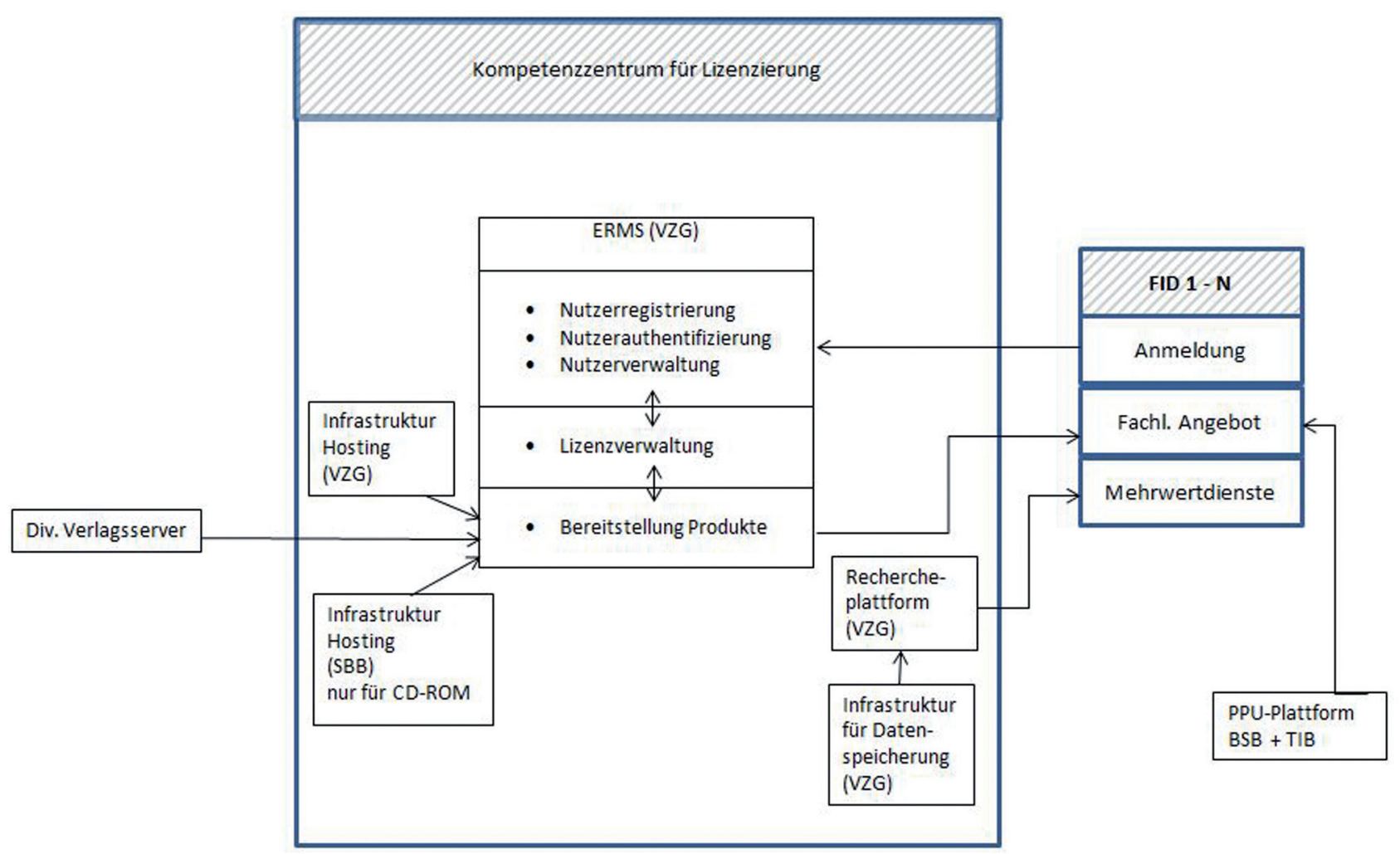

Abb. 2: Infrastrukturkonzept des Kompetenzzentrums für Lizenzierung (KfL)

\section{FID-Lizenzen}

Mit den DFG-geförderten „FID-Lizenzen“ wurde im Rahmen der überregionalen Lizenzierung kostenpflichtiger elektronischer Medien ein neuer Lizenztyp definiert, der komplementär zu den hier bereits etablierten Formen hinzutritt. ${ }^{15}$ Die FID-Lizenzen bauen auf Erfahrungen mit den DFG-geförderten National- und Allianz-Lizenzen sowie den an der Staatsbibliothek zu Berlin entwickelten Lizenzen für die Virtuelle Fachbibliothek des Sondersammelgebiets Ost- und Südostasien (CrossAsia) auf, die für den speziellen Bedarfskontext der FID angepasst wurden. ${ }^{16}$ Sie

15 Vgl. Schäffler, Hildegard: Elektronische Medien in der überregionalen Literaturversorgung: Nationallizenzen, Allianzlizenzen, FIDLizenzen. In: Göttker, Susanne; Wein, Franziska (Hrsg.): Neue Formen der Erwerbung. Berlin 2014 (Bibliotheks- und Informationspraxis; 47) sowie Schäffler, Hildegard: (Über-)regionale Lizenzierung in Deutschland: National- und Allianz-Lizenzen, GASCO-Konsortien, FID-Lizenzen. Bibliothekartag 2015. Vortragsfolien: http://opus4.kob v.de/opus4-bib-info/files/1671/Workshop_FID-Lizenzen_Schaeffler_f inal-2.pdf.

16 Die Virtuelle Fachbibliothek CrossAsia an der Staatsbibliothek zu Berlin bietet Einzelnutzern in Deutschland seit Jahren den zentralen Zugang zu lizenzpflichtigen digitalen Medien mit Fachbezug zu Ostund Südostasien. Vgl. http://crossasia.org/ressourcen/database search.html. Zur Abgrenzung FID- und Allianz-Lizenzen vgl. Lipp, zielen auf die Bereitstellung für einen fachlich definierten Nutzerkreis in Deutschland ab - in der Regel handelt es sich dabei um eine vom jeweiligen FID definierte Anzahl von Einzelnutzern mit institutioneller Anbindung. Für diese campusunabhängige Nutzerkreisbestimmung gibt es Vorbilder bei den CrossAsia-Lizenzen, während alle übrigen Lizenztypen in der überregionalen Versorgung institutioneller Nutzer mit elektronischen Medien campusweit verfügbar gemacht werden (Campuslizenzen). Es handelt sich bei den FID-Lizenzen daher um ein völlig neues, unter rein fachlichen Aspekten entwickeltes Konstrukt.

Für die FID-Lizenzen sind im Rahmen des DFG-Förderprogramms „Fachinformationsdienste für die Wissenschaft“ sowie der „Grundsätze für den Erwerb von Publikationen im DFG-geförderten System der Fachinformationsdienste für die Wissenschaft“ (FID-Grundsätze) Standards festgelegt worden, die in den wesentlichen Anforderungen (Datenlieferungen, Rechte zum Hosting und Archiving, zum Aufbau von Mehrwertdiensten, Open-

Anne: FID-Lizenzen und Allianz-Lizenzen: Zielsetzungen und Unterschiede. Bibliothekartag 2015. Vortragsfolien: https://opus4.kobv. de/opus4-bib-info/files/1671/Allianz_FID_Lizenzen_Lipp.pdf. 
Access-Klausel) den Vorgaben für die Allianz-Lizenzen folgen. ${ }^{17}$ Die Finanzierung der FID-Lizenzen erfolgt anteilig durch die DFG, die 2/3 der Lizenzkosten trägt, und die FID, die jeweils 1/3 der Lizenzkosten in Eigenleistung erbringen müssen. Hervorzuheben ist zudem die Tatsache, dass die FID-Lizenzen anders als die National- und Allianz-Lizenzen ausschließlich auf die Bedienung eines von der jeweiligen Fachcommunity formulierten Bedarfes ausgerichtet sind und einem direkten Versorgungsauftrag unterliegen. Zielsetzung ist die bedarfsorientierte Versorgung von Fachcommunities mit Ressourcen des „Spitzenbedarfes“, ergänzend zum bereits verfügbaren Angebot von Hochschulen und Forschungseinrichtungen. FID-Lizenzen können für alle Produkttypen abgeschlossen werden, im Zentrum des Interesses stehen hier jedoch Produkte von mittlerer bis geringer Marktdurchdringung (Nischenprodukte).

Die zentrale Herausforderung bei der Etablierung von FID-Lizenzen liegt in der Findung von Lizenz- und Geschäftsmodellen sowie in der Schaffung von geeigneten organisatorischen und technischen Voraussetzungen für die damit verbundenen Anforderungen der überregionalen Bereitstellung.

\section{Verhandlung von FID-Lizenzen ${ }^{18}$}

Die Verhandlung von FID-Lizenzen durch das Kompetenzzentrum wird von den FID angestoßen, die die zu verhandelnden Produkte auswählen und die entsprechenden fachspezifischen Nutzerkreise definieren. Häufig verbinden die FID diese Auftragserteilung an das KfL auch mit Vorgaben hinsichtlich gewünschter Geschäftsmodelle sowie des finanziellen Rahmens, die mit den Anforderungen der DFG-Förderkriterien (FID-Grundsätze) und mit den Möglichkeiten der Anbieter überein zu bringen sind. FIDLizenzen müssen bedarfsorientiert und nutzerkreisadäquat gestaltet werden, sie sollen zu realistischen Kosten erworben werden und mit vertretbarem Aufwand adminis-

17 Vgl. Merkblatt 12.101: Grundsätze für den Erwerb von Publikationen im DFG-geförderten System der Fachinformationsdienste für die Wissenschaft. Deutsche Forschungsgemeinschaft 2012: http://www.d fg.de/formulare/12_101.

18 Vgl. Hillenkötter, Kristine: FID-Lizenzen in die Praxis bringen: Anforderungen und Herausforderungen. Bibliothekartag 2015. Vortragsfolien: https://opus4.kobv.de/opus4-bib-info/files/1671/Biblioth ekartag+2015_Vortrag_Kompetenzzentrum_Lizenzierung_Hi_2605 2015.pdf. trierbar sein. Gleichzeitig ist die Verhandlung von FIDLizenzen auch in Relation zu anderen Lizenzierungskontexten im Rahmen der etablierten Beschaffungsstrukturen für die überregionale Lizenzierung elektronischer Medien zu betrachten.

\subsection{Nutzerkreismodelle der FID}

Die einzelnen Nutzerkreismodelle der FID können je nach Fachcommunity sehr unterschiedlich und beliebig komplex gestaltet sein. Überwiegend wird die Freischaltung der Lizenz für den gesamten fachlich definierten Nutzerkreis gewünscht. Diese Lösung stellt derzeit die Standardlösung dar. Dafür sind drei Grundmodelle in unterschiedlicher Ausprägung zu beschreiben:

- Spezifischer Nutzerkreis: Liste von Institutionen + Anzahl daraus zu erwartender Einzelnutzer (mit definierter Institutionszugehörigkeit)

- Virtueller Nutzerkreis: Virtuelle Institution „FID X bzw. Fachgesellschaft X“ + Anzahl darauf bezogener Einzelnutzer (ohne definierte Institutionszugehörigkeit)

- Nationallizenz: Alle wissenschaftlichen Einrichtungen in Deutschland (keine Einschränkung der Nutzergruppe)

Daneben werden von den FID auch produktspezifische Nutzerkreise in den Blick genommen:

- In Abhängigkeit von der (kooperativen) Finanzierung der Lizenzkosten.

- In Abhängigkeit von der fachlichen Relevanz des Produktes für den gesamten Nutzerkreis.

Produktspezifische Nutzerkreismodelle stellen komplexere Anforderungen an die Lizenz-, Geschäfts- und Bereitstellungsmodelle für die betreffenden FID-Lizenzen und auch an die damit verbundenen Workflows. Das KfL hat sich daher während der Aufbauphase der internen Betriebsstrukturen zunächst auf die Realisierung der oben beschriebenen Standardlösung beschränkt. Die Befassung mit den Anforderungen produktspezifischer Nutzerkreismodelle wird in einem zweiten Schritt erfolgen (s. u. unter 4.2).

\subsection{Lizenz- und Geschäftsmodelle für FID- Lizenzen}

Die Entwicklung von Lizenz- und Geschäftsmodellen für FID-Lizenzen steht in enger Relation zu den Anforderun- 
gen der FID-Grundsätze ${ }^{19}$ und den Nutzerkreismodellen der FID. Gleichzeitig kann hier auf bereits vorhandene Lizenzmodelle zurückgegriffen werden, die dann für den FID-Kontext angepasst werden müssen (Nationallizenz, Allianz-Lizenz/Opt-In-Konsortium, lokale Campuslizenz). In den bisherigen Verhandlungen haben sich die folgenden Lizenzmodelle bewährt:

- FIDNutzerkreis: FID-Lizenz für den definierten Nutzerkreis. Die Ausgestaltung erfolgt in Abhängigkeit vom Nutzerkreismodell, zum Teil wird die FID-Lizenz mit einer lokalen Campuslizenz für die FID-Bibliothek kombiniert.

- FIDCampus: FID-Lizenz zur campusweiten Freischaltung für alle Institutionen eines definierten Nutzerkreises, nach dem Vorbild der herkömmlichen Campuslizenzen.

- FIDNationallizenz: Klassische Nationallizenz ohne (fachliche) Eingrenzung der Nutzergruppe, nach dem Vorbild der DFG-geförderten Nationallizenzen.

Dabei stellt die Verwendung der Modelle FIDCampus und FIDNationallizenz die Ausnahme dar: Über $80 \%$ aller bislang bearbeiteten Verhandlungsaufträge entfallen auf das Modell FIDNutzerkreis.

Die Lizenzmodelle werden mit den bekannten, insbesondere bedarfsorientierten Geschäftsmodellen kombiniert (Kauf, Subskription, Pick \& Choose, PDA, EBS, PPU/PPV, Kontingente/Token), wobei sich die Wahl der Geschäftsmodelle an den Produktwünschen und Präferenzen der FID sowie den Möglichkeiten der Anbieter orientiert.

Neben den genannten Formen sind auch Lizenzmodelle für produktspezifische Nutzerkreise für die FID von Interesse:

- FIDOpt-In: Kostenbeteiligung durch (einzelne) Institutionen des Nutzerkreises und Beschränkung der Freischaltung auf diese Institutionen, nach dem Vorbild der Konsortial- bzw. Allianz-Lizenzen.

- FIDSelected: Fachliche Relevanz und Freischaltung nur für einen Teil des definierten Nutzerkreises, als Spielart von FIDNutzerkreis.

Das Kompetenzzentrum wird die Verhandlung dieser Modelle aufnehmen, sobald die technischen Voraussetzungen für die Bereitstellung entsprechender Lizenzen gegeben sind (s. u. unter 5.2).

19 Vgl. Merkblatt 12.101: Grundsätze für den Erwerb von Publikationen im DFG-geförderten System der Fachinformationsdienste für die Wissenschaft. Deutsche Forschungsgemeinschaft 2012: http://www.d fg.de/formulare/12_101.
Die Preisgestaltung für die FID-Lizenzen befindet sich noch im experimentellen Stadium. Bezugsgrößen und Parameter der Preisbildung sind der Listenpreis für eine Campuslizenz, die Verbreitung des Produktes in Deutschland und die Anzahl definierter Einzelnutzer im FID-Nutzerkreis. Derzeit herrschen hier zwei Modellvarianten vor:

- Preis pro Produkt: Listenpreis Campuslizenz/Paketpreis, ggf. in Relation zum Verbreitungsgrad in Deutschland (analog zu dem Verfahren der Preisbildung bei den Nationallizenzen).

- Preis pro Einzelnutzer: Listenpreis Campuslizenz in Relation zur Anzahl der Nutzer.

Verschiedene Mechanismen zur Kostendämpfung sind derzeit noch in der Erprobung.

Die Modellentwicklung für FID-Lizenzen ist noch nicht abgeschlossen und orientiert sich weiterhin an den Vorgaben der FID, den Möglichkeiten der Anbieter sowie ggf. weiterer $\mathrm{zu}$ berücksichtigender Rahmenbedingungen. Im Zentrum der Bemühungen steht dabei die weitere Ausgestaltung des Modells FIDNutzerkreis sowie der Modelle FIDOpt-In und FIDSelected.

\subsection{Interdependenzen zwischen FID-Lizenzen und bestehenden Beschaffungsstrukturen}

Bei der Bearbeitung von Verhandlungsaufträgen der FID wird regelmäßig überprüft, ob für das gewünschte Produkt bereits andere überregionale Abschlüsse im Rahmen der bestehenden Konsortialstrukturen existieren und wie sich eine potentielle FID-Lizenz dazu ggf. in Relation setzen könnte. Herausforderungen ergeben sich dabei aus der Tatsache, dass sich die FID-Lizenzen hinsichtlich der Nutzerkreise, Konditionen und Organisationsstrukturen von den Konsortiallizenzen signifikant unterscheiden, wobei die verwendeten Lizenzmodelle in der Regel auf gemeinsame Grundlagen zurückzuführen sind. Parallelangebote von Konsortien und FID-Lizenzen stellen Institutionen, die am betreffenden Konsortium teilnehmen, aber auch wesentliche core user mit Berechtigung für die parallele FIDLizenz aufweisen, vor Wahlmöglichkeiten und bergen damit das Potential zur Schwächung der betreffenden Konsortien - aus denen sich die Institutionen ggf. zurückziehen, wenn sie ihre core user durch das (noch) kostenfreie FID-Angebot bedient wissen.

Besonders betroffen können Zeitschriftenkonsortien sein, die auf diese Weise nicht nur Teilnehmer, sondern auch Inhalte verlieren können, sofern sie z. B. nach dem 
holdingsbasierten Lizenzmodell mit Cross-Access-Option angelegt sind. In allen Fällen bedeutet der Verlust von Teilnehmern für die Konsortien in der Regel aber den Verlust von Rabattierungen und damit einen Anstieg der Kosten für die verbleibenden Teilnehmer.

Da die FID-Lizenzen jedoch auf den Spitzenbedarf einzelner Fachcommunities ausgerichtet sind und sich auf „Nischenprodukte“ mit mittlerer bis niedriger Marktdurchdringung fokussieren, sind Überschneidungen mit den eher der Bedienung des Grundbedarfes von Hochschulen und Forschungseinrichtungen verhafteten Angeboten der Konsortien sehr selten.

Dennoch gilt es vor diesem Hintergrund im Bedarfsfall Lösungen zu entwickeln, die ein sinnvolles, komplementäres Nebeneinander von etablierten Konsortial- und neuen FID-Lizenzen ermöglichen.

Denkbar wäre hier insbesondere bei Zeitschriftenliteratur die Entwicklung von Lizenzmodellen für FID-Lizenzen in Korrelation zu bestehenden Konsortialabschlüssen, z. B. als Upgrade der Konsortiallizenz für den FID-Nutzerkreis und die FID-Konditionen. In Frage kommen dafür Angebote im Rahmen von (über-)regionalen Konsortien ohne DFG-Förderung - überregionale Angebote der Allianz-Lizenzen sind aufgrund der potentiellen Doppelförderung hier ausgenommen. Hinsichtlich der Datenbanken und E-Book-Angebote wird die Interdependenz zwischen den unterschiedlichen Lizenzierungskontexten voraussichtlich einfacher zu lösen sein. Hier sind ergänzend $\mathrm{zu}$ bestehenden (über)regionalen Lizenzen eher separate und individuelle Parallellösungen für FID-Lizenzen denkbar, die mit den Verlagen gemeinsam zu finden sind.

Das Kompetenzzentrum nimmt bei Bedarf mit den betroffenen Konsortien Kontakt auf und sucht hier in gegenseitiger Abstimmung nach Verfahrenswegen. Sofern eine Verknüpfung zwischen FID- und Konsortiallizenzen sinnvoll erscheint, wird das KfL in Absprache mit den betroffenen Konsortialführern entsprechende Modelle entwickeln. In der Verhandlungspraxis sind diese Fälle bislang jedoch noch nicht aufgetreten.

Über die Website des Kompetenzzentrums ist eine Liste aller bislang abgeschlossenen FID-Lizenzen einsehbar, die über die Plattform des KfL bereitgestellt werden. ${ }^{20} \mathrm{Da}$ rüber hinaus informiert das KfL regelmäßig die Konsortien im Rahmen der GASCO ${ }^{21}$ über seine Aktivitäten.

20 Vgl. http://www.fid-lizenzen.de/angebote.

21 Vgl. GASCO (German, Austrian, and Swiss Consortia Organisation) - Arbeitsgemeinschaft Deutscher, Österreichischer und Schweizer Konsortien: https://www.hbz-nrw.de/angebote/digitale_inhalte/ gasco/.

\subsection{Umsetzbarkeit der Förderkriterien}

Die DFG knüpft die anteilige Finanzierung der FID-Lizenzen an definierte Standards und Konditionen, die die FIDLizenzen gewährleisten müssen (FID-Grundsätze). ${ }^{22}$ Die Anforderungen ähneln denen für die DFG-geförderten Allianz-Lizenzen, auf deren Grundlage sie entwickelt wurden. Zentrale Anforderungen sind u. a. die physische Auslieferung des Produktes (Lieferung von Meta- und Inhaltsdaten) sowie erweiterte dauerhafte Nutzungsrechte wie das Recht auf lokales Hosting, das Recht zum Aufbau von Mehrwertdiensten auf Basis der ausgelieferten Daten (z. B. Volltextindexierung, Text and Data Mining) und eine Open-Access-Klausel bei Zeitschriftenliteratur (OAgrün). Ausnahmen von diesen Vorgaben sind grundsätzlich möglich, müssen vom FID jedoch explizit fachlich begründet werden. Die Verantwortung für die Verausgabung von Fördermitteln für FID-Lizenzen, die nicht den Vorgaben der Förderkriterien folgen, trägt der FID.

Ähnlich wie bereits aus dem Kontext der Allianz-Lizenzen bekannt, erfordert die Verhandlung der Förderkriterien von FID-Lizenzen einen hohen Aufwand in der Kommunikation zwischen Kompetenzzentrum und Anbieter, sofern der Verlag nicht bereits aus anderen DFGgeförderten Lizenzierungskontexten mit diesen Anforderungen vertraut ist. Häufig sehen die Anbieter in den Anforderungen eine Konkurrenz zu den Verlagsaktivitäten. Besonders schwierig gestaltet sich das damit skizzierte Spannungsfeld zwischen den Bedarfen der FID, den Anforderungen der FID-Grundsätze und den Möglichkeiten der Anbieter bei Aggregatorprodukten, die häufig von den FID für die Verhandlungen nominiert werden, jedoch in der Regel die Förderkriterien nicht erfüllen können, da die betreffenden Anbieter nicht über die Rechte der bereitgestellten Inhalte verfügen (s.u. unter 4.5.1).

\subsection{Werkstattbericht: Bearbeitung von Verhandlungsaufträgen der FID ${ }^{23}$}

Im ersten Projektjahr wurde das KfL für neun FID aktiv, die im Jahr 2014 einen FID-Antrag stellten und von denen vier

22 Vgl. Merkblatt 12.101: Grundsätze für den Erwerb von Publikationen im DFG-geförderten System der Fachinformationsdienste für die Wissenschaft. Deutsche Forschungsgemeinschaft 2012: http://www.d fg.de/formulare/12_101.

23 Vgl. Stanek, Ursula: Verhandlung von FID-Lizenzen durch das Kompetenzzentrum für Lizenzierung - Statusbericht. Bibliothekartag 2015. Vortragsfolien: https://opus4.kobv.de/opus4-bib-info/files/167 
von der DFG bewilligt wurden. Ergänzend wandten sich drei FID mit Verhandlungsaufträgen an das KfL, die bereits in der ersten Antragsrunde 2013 bewilligt worden waren. 2015 stellten 13 in der Antragsphase befindliche FID Verhandlungsaufträge an das KfL. Fachlich lag im ersten Projektjahr der Schwerpunkt im naturwissenschaftlichen Bereich, im zweiten im geisteswissenschaftlichen Bereich, der auch regionale Sondersammelgebiete mit einschloss.

Am Beginn der Kooperation mit einem FID steht die Kontaktaufnahme des FID mit dem KfL, in der Regel per EMail. Das entsprechende Verhandlungsteam (SUB Göttingen oder SBB) klärt in der Folge die zentralen Rahmenbedingungen der gewünschten Dienstleistungen. Hierbei handelte es sich bisher überwiegend um Verhandlungsaufträge. Schwerpunkte der ersten Beratung sind insbesondere die Definition der FID-spezifischen Nutzergruppe sowie die gewünschten Produkte an sich, für die z.B. auch Bestandsanalysen durchgeführt werden. Einige Produkte werden schon in diesem Stadium aus dem Verhandlungsauftrag entfernt, z. B. weil sie bereits weit verbreitet sind oder nicht dem Spitzenbedarf zuzurechnen sind. Nach der Schärfung des Verhandlungsauftrags erfolgt die Kontaktaufnahme durch das KfL mit den jeweiligen Anbietern, ggf. auch die Information an betroffene Konsortien. Da FID-Lizenzen nicht an einen bestimmten Campus gebunden sind, sondern überregional bereitgestellt werden, entsprechen sie nicht den üblichen Lizenzmodellen der Anbieter. Und auch wenn das Lizenzmodell und die DFGRahmenbedingungen an sich schon bekannt waren, stellt doch fast jede Bitte um Angebotserstellung Neuland für die Anbieter dar, da jeder FID Besonderheiten in der Definition der eigenen Nutzergruppe vorsieht, die individuell zu bewerten und zu kalkulieren sind.

\subsubsection{Produktanalyse}

Durch den naturwissenschaftlichen Fokus der Verhandlungsaufträge 2014 waren vor allem Produkte der großen, international agierenden STM-Verlage betroffen, die aber meist mit Hinweis auf die etablierten Konsortialstrukturen, befürchtete Umsatzverluste oder langwierige interne $\mathrm{Ab}$ stimmungsprozesse keine bzw. keine finanziell vertretbaren Angebote abgaben. Das andere Extrem stellten die

1/Stanek_26_Mai_2015_St.Petersburg.pdf sowie Stanek, Ursula; Hillenkötter, Kristine: Verhandlung von FID-Lizenzen durch das Kompetenzzentrum für Lizenzierung - Statusbericht. In: o-bib. Das offene Bibliotheksjournal 2(4) (2015), Tagungsband zum Bibliothekartag 2015, S. 95-107, http://dx.doi.org/10.5282/o-bib/2015H4S95-107.
Fachgesellschaften dar, die oft nicht einmal auf die Anfrage hinsichtlich der Benennung des richtigen Ansprechpartners für ein Angebot reagierten. Am kooperativsten agierten die mittelgroßen Anbieter, deren Produkte über eine relativ geringe Marktdurchdringung verfügten.

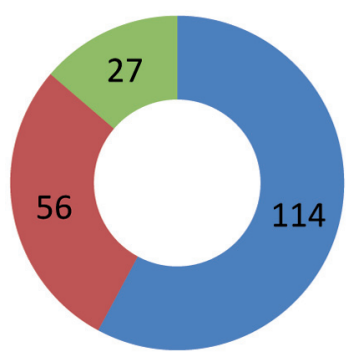

Zeitschriften-
Produkte
Datenbank-
Produkte
E-Book-
Produkte

Abb. 3: Anzahl der Produkte und ihre Verteilung nach Produkttypen (Jan. 2014 - Mai 2015)

Zwischen Januar 2014 und Mai 2015 wurden Verhandlungsaufträge für 197 Produkte an das KfL gerichtet (vgl. Abb.3). Bei mehr als der Hälfte handelte es sich um Zeitschriftenprodukte, gut ein Viertel der Verhandlungsaufträge betrafen Datenbanken, sowohl Volltext- als auch Bibliographische Datenbanken. Die kleinste Gruppe bildeten bislang E-Book-Produkte. Bezogen auf die bisherige Projektlaufzeit ergibt sich insofern hinsichtlich der Produkttypen ein recht ausgeglichenes Bild. Betrachtet man die beiden Verhandlungsjahre jedoch getrennt, lässt sich im Jahr 2014 ein deutlicher Schwerpunkt bei den Zeitschriften feststellen (76 Produkte, davon bei $75 \%$ Einzeltitel). Erst 2015 traten in nennenswertem Umfang auch andere Produkttypen hinzu.

Einen Sonderfall stellten 2015 Aggregatorprodukte dar, die fast ein Drittel der Verhandlungsaufträge ausmachten, wobei es sich sowohl um Zeitschriften- als auch insbesondere um E-Book-Aggregatoren handelte. Aggregatorprodukte bieten für FID in vielerlei Hinsicht große Vorteile: Mit einem einzigen Produkt werden mehrere Ressourcen gebündelt, die zudem potentiell auch von einer Vielzahl von Verlagen stammen. Mit der Einbindung eines solchen Produkts in sein Portfolio kann ein FID daher mit einer einzigen Lizenz eine bedeutende Anzahl unterschiedlicher Ressourcen anbieten. Der Nachteil der Aggregatoren ist jedoch, dass sie sehr oft nicht die Anforderungen der DFG für FID-Lizenzen erfüllen können. Dies betrifft insbesondere die Rechte hinsichtlich des Archivierens und des Hostings, da die Aggregatoren selbst nicht über diese verfügen, sondern sie nur vom Verlag eingeräumt werden können. Durch die in den DFG-Förderrichtlinien vorgesehene Option, in fachlich begründeten Fällen Ausnahmen zuzulassen, erhielt das 
KfL 2015 dennoch zahlreiche Verhandlungsaufträge für Aggregatorprodukte.

In der Praxis waren die Erfahrungen mit den Aggregatoren recht unterschiedlich. Auf der einen Seite handelte es sich bei den gewünschten Produkten um Aggregatoren, wie z.B. Casalini, die mit ihrer Plattform Torrossa für eine Vielzahl italienischer Verlage, die über keine eigene Online-Plattform verfügen, als Dienstleister fungieren. Hier war die Erfüllung der DFG-Anforderungen meist kein Problem. Auf der anderen Seite standen große Volltextdatenbanken beispielsweise von Ebscohost, die die für die FID relevanten Rechte nicht einräumen können (z.B. keine Archiv- und Hostingrechte, keine Datenlieferung und damit oft auch keine Mehrwertdienste).

\subsubsection{Nutzerkreise, Lizenz- und Geschäftsmodelle}

Hinsichtlich der unterschiedlichen Nutzerkreisdefinitionen gestalten sich die Erfahrungen in den Verhandlungen sehr unterschiedlich:

- Spezifischer Nutzerkreis: Der FID benennt eine Liste von Institutionen oder Instituten, aus denen die berechtigten Nutzer kommen. Es hat sich gezeigt, dass die Anbieter mit dieser Nutzergruppendefinition gut umgehen, vor allem wenn zudem die erwartete Anzahl berechtigter Nutzer sowie die Kriterien der Berechtigung vorgegeben werden (z. B. nur Professoren, wissenschaftlicher Mittelbau etc.). Dieses Nutzerkreismodell kann bis hin zu campusweiten Lizenzen gehen, wobei in diesen Fällen die Finanzierbarkeit alleine durch den FID oft nicht möglich ist.

- Virtueller Nutzerkreis: Die berechtigten FID-Nutzer können keiner bestimmten Institution zugeordnet werden. Bei diesem Modell wird z.B. die FID-Community mit der Mitgliedschaft in einer Fachgesellschaft verknüpft bzw. der FID fungiert selbst als eine Art Fachgesellschaft. Da für die Anbieter die Anzahl der bestehenden Lizenzen und der jeweiligen Lizenzierungskontexte (lokale Einzellizenzen, konsortiale $\mathrm{Pa}$ ketlizenzen etc.) eine Rolle spielt, ist die Fachcommunity bei dieser Definition für die Anbieter praktisch nicht greifbar, was eine Kalkulation in der Regel kaum möglich macht.

- Nationallizenz: Alle Nutzer aus wissenschaftlichen Einrichtungen in Deutschland sind berechtigt, auf die Inhalte der FID-Lizenz zuzugreifen. Da sehr viele Anbieter die Nationallizenzen bereits kennen oder schon Nationallizenzen abgeschlossen haben, ist diese Nutzergruppendefinition den meisten Anbietern gut zu vermitteln. Durch die Größe der potentiellen Nutzer- gruppe ist dieses Modell jedoch nur in wenigen Fällen umsetzbar, da es nur selten zu finanziell vertretbaren Angeboten kommt.

Wie eben dargestellt, hat die Definition und Größe der jeweiligen Nutzergruppe eines FID großen Einfluss darauf, ob ein attraktives Angebot abgegeben wird. Weitere zentrale Parameter hierfür sind jedoch auch die gewünschten Lizenz- bzw. Geschäftsmodelle. Manche FID äußern bereits bei der Auftragserteilung sehr konkrete Wünsche, zum Teil müssen sich KfL und FID nach den von den Anbietern angebotenen Modellen richten. Für alle Beteiligten stellen die Lizenzmodelle des Kaufs (v. a. bei Datenbanken oder Archiven) oder der Subskription (v.a. bei laufenden Zeitschriften) den üblichen Fall dar. Das Lizenzmodell der Nationallizenz kann ebenfalls durchaus als etabliert bezeichnet werden, auch wenn die Anzahl der erfolgreich verhandelten FID-Lizenzen nach diesem Modell gering ist. Besonders interessant für den FID-Kontext sind jedoch nutzungs- und bedarfsorientierte Modelle, bei denen der Pool an potentiell nutzbaren Ressourcen deutlich größer ist als dies im Rahmen einer konventionellen Lizenz der Fall wäre. Die Mitglieder der Fachcommunity können in diesem größeren Datenpool suchen und die für sie relevanten Ressourcen nutzen. Damit entsprechen bedarfs- oder nutzungsbasierte Lizenzmodelle in besonderem Maße den Anforderungen der DFG.

Abhängig vom Produkttyp sind unterschiedliche Umsetzungen möglich. Im Zeitschriftenbereich können nutzungsbasierte Modelle beispielsweise durch den Kauf von Artikelkontingenten realisiert werden. Auf diese Weise kann ein FID ein großes Angebot an Inhalten für einen überschaubaren Preis anbieten. Bezahlt werden nur jene Artikel, die von der FID-Community auch genutzt werden. Hier stellen sich jedoch einige Fragen, z. B. wie flexibel in dem Lizenzmodell auf sehr große bzw. sehr geringe Nachfrage reagiert wird oder wie sich die DFG-Anforderungen im Falle solcher Kontingentmodelle umsetzen lassen. In der Verhandlungsrunde 2015 wurden derartige Lizenzen beim KfL erstmals in Auftrag gegeben und konnten auch erfolgreich verhandelt werden. Der Praxistest kann erst erfolgen, wenn die Bewilligungen der betreffenden FIDAnträge vorliegen und die entsprechenden Lizenzen abgeschlossen wurden.

Im E-Book-Bereich hat sich die nutzerorientierte Erwerbung - oder „patron driven acquisition“ (PDA) bzw. „demand driven acquisition“ (DDA) - in den letzten Jahren erfolgreich etabliert, man unterscheidet zwischen Aggregatorangeboten und verlagsbasierten Angeboten. Für die E-Book-Aggregatoren gilt das bereits oben Gesagte, dass die DFG-Anforderungen in der Regel nicht erfüllt werden 
können (wobei dies nicht zwingend einen Verhandlungsabbruch bedeuten muss). Diese Hürde nehmen demgegenüber die verlagsbasierten Angebote, da deren Anbieter über die erforderlichen Rechte verfügen. Hier stellt sich jedoch die Frage, ob der Verlag über eine ausreichende Menge fachlich relevanter E-Books verfügt, sodass das verlagsspezifische PDA-Modell finanziell attraktiv umgesetzt werden kann.

\subsubsection{Verhandlungsergebnisse}

Für mehr als ein Drittel der eingereichten Verhandlungsaufträge (73 von 197) konnten die Verhandlungen von FIDLizenzen zu einem positiven Ergebnis gebracht werden. Dies bedeutet, dass ein Angebot zustande kam und der Verhandlungsspielraum so weit wie möglich zu Gunsten des FID ausgereizt wurde - es bedeutet leider nicht, dass diese Angebote vom FID auch zu finanzieren wären. Schlussendlich entscheidet auch der Ausgang des Begutachtungsverfahrens der DFG über die Umsetzung der Verhandlungsergebnisse: Da das KfL meist im Vorfeld der Antragstellung eines FID aktiv wird, sind im Falle einer Ablehnung des Antrags durch die DFG auch die Verhandlungsergebnisse des KfL hinfällig.

\section{Bereitstellung von FID-Lizenzen}

Die Bereitstellung der FID-Lizenzen erfolgt durch das KfL, anschließend informiert der betreffende FID seinen Nutzerkreis über die neuen Angebote und lädt seine Nutzer ein, sich für die Lizenzen anzumelden.

Bereits im Mai bzw. Juni 2015 konnten die ersten durch das KfL verhandelten FID-Lizenzen, deren Lizenzmodell eine Campus-Freischaltung der berechtigten Einrichtungen umfasste bzw. dem Nationallizenz-Modell folgten, freigeschaltet und den Wissenschaftlern zur Verfügung gestellt werden. Es handelte sich um Zeitschriften für den FID Mathematik und Zeitschriften für den FID Erziehungswissenschaften und Bildungsforschung. Über $90 \%$ der derzeit über das KfL abgeschlossenen FID-Lizenzen werden jedoch seit September 2015 über die Bereitstellungsplattform des KfL zugänglich gemacht. ${ }^{24}$

24 Vgl. http://www.fid-lizenzen.de/angebote.

\subsection{Bereitstellungsplattform des Kompetenzzentrums (KfL-ERMS) ${ }^{25}$}

Die Bereitstellungsplattform des KfL (KfL-ERMS) wurde im Rahmen der Projektarbeit entwickelt. Dieses System ermöglicht derzeit die Bereitstellung von FID-Lizenzen nach dem Modell FIDNutzerkreis - perspektivisch werden hier auch produktspezifische Lizenzmodelle wie FIDOpt-In oder FIDSelected unterstützt werden. Die Plattform dient der Anmeldung und Verwaltung der Nutzer, der Pflege der Produkteinträge und gewährleistet die Authentifizierung für FID-Lizenzen. Das KfL-ERMS bildet das Herzstück der technischen Infrastruktur des Kompetenzzentrums und konnte am 1. September 2015 in Betrieb genommen werden.

\subsubsection{Aufgaben und Ziele}

Das System musste drei Anforderungen gerecht werden:

1. Es wird ein Electronic Ressource Management System (ERMS) für die FID benötigt. Das ERMS verwaltet die Produkte der FID und dazugehörigen Lizenzmodelle und ist mit einem Workflow-System kombiniert, um bei der Verwaltung optimal zu unterstützen.

2. Es sollte ein Präsentationsmodul für vom FID lizenzierte Produkte entwickelt werden. Darüber hinaus können FID mit Hilfe dieses Moduls ihren kompletten eigenen Web-Auftritt verwirklichen.

3. Die Entwicklung eines Moduls für die Zugangsteuerung ist erforderlich und von zentraler Bedeutung. Denn die FID-Lizenzen werden für spezifische Nutzergruppen erworben. Daher muss zu jedem Zeitpunkt sichergestellt sein, dass ausschließlich berechtigte Nutzer Zugang zu den für sie lizenzierten Produkten erhalten.

Die drei Grundaufgaben wurden als One-Stop-System für FID, deren Nutzer und für das KfL selbst realisiert. Der Vorteil dieses Konzepts für FID-Nutzer liegt auf der Hand. Die Nutzer können sich von der initialen Registrierung bis zur Verwendung einer für sie bestimmten elektronischen Ressource immer innerhalb der Homepage des zuständigen FID bewegen. Interdisziplinär arbeitende Nutzer, die berechtigt sind, verschiedenen FID anzugehören, finden bei jedem FID immer dieselben Bedienkonzepte vor. Dazu

25 Vgl. Steilen, Gerald: Technische Infrastruktur des Kompetenzzentrums für Lizenzierung zur Bereitstellung von FID-Lizenzen. Technische Realisierung. Bibliothekartag 2015. Vortragsfolien: https://op us4.kobv.de/opus4-bib-info/files/1671/steilen_2015-05-26_02.pdf. 
gehört selbstverständlich, dass solche Nutzer mit einer einzigen digitalen Identität ihre von verschiedenen FID stammenden Rechte akkumulieren können. Natürlich profitieren die einzelnen FID auch von diesem Konzept. Das KfL kann ihnen auf Wunsch die komplette technische Infrastruktur zum Betrieb eines FID als Dienstleistung effizient anbieten und sie von Routineaufgaben nachhaltig entlasten. Die Serviceteams des KfL profitieren durch erhebliche Arbeitserleichterung von diesem Konzept. Denn sie werden durch automatisierte Arbeitsabläufe, auch in ihrer Kommunikation mit den FID, unterstützt und verfügen sowohl immer über einen umfassenden Überblick als auch über die notwendigen Detailinformationen während sämtlicher Phasen der Lizenz- und Nutzerverwaltung.

Die Zugangssteuerung für FID-Nutzer zu lizenzierten Produkten wird technisch über Proxies geregelt. Als Proxies werden Komponenten in einem Rechnernetzwerk bezeichnet, die als Vermittler zwischen verschiedenen Rechnern arbeiten. Auf der einen Seite nehmen Proxies Anfragen entgegen und leiten diese an die eigentlichen Zielsysteme weiter. Auf der anderen Seite können Proxies als Vermittler zusätzlich verschiedene Prüfungen vornehmen. Im Rahmen des KfL ist es Aufgabe hoch spezialisierter Proxies zu überprüfen, ob die Anfrage überhaupt zum Ziel durchgestellt werden darf, also, ob für die Erfüllung der Anfrage die notwendigen Rechte bei einem FID-Nutzer vorliegen. Die Berechtigung selbst können die eingesetzten Proxies an zwei Merkmalen feststellen. Am wichtigsten ist die Feststellung der individuellen Nutzungsrechte eines FID-Nutzers. Liegt ein Nutzungsrecht für eine bestimmte elektronische Ressource vor, so stellt ein Proxy die technisch notwendige Verbindung zwischen Anfragendem und dem Zielsystem her. Die vom KfL eingesetzte ProxyTechnik beherrscht hierzu ein international praktiziertes Verfahren namens Shibboleth. Mit Hilfe von Shibboleth werden gleichzeitig drei Ziele erreicht. Das erste besteht im Komfort. Berechtigte Nutzer müssen sich bei ihrem FID nur ein einziges Mal erfolgreich anmelden, auch dann, wenn sie von einer geschützten Ressource, wie z.B. einem bestimmten Angebot des Verlags A, zu einer anderen geschützten Ressource, beispielsweise einem bestimmten Angebot des Verlags B, wechseln. Die komplette technische Kommunikation der verschiedenen Systeme zur Authentifizierung und Autorisierung geschieht ohne $\mathrm{Zu}$ tun des Nutzers vollständig automatisch im Hintergrund. Dieses Verfahren wird als Single Sign-On bezeichnet. Das zweite Ziel besteht in der Nutzungsmöglichkeit elektronischer Ressourcen unabhängig vom geographischen Aufenthaltsort des Nutzers, der technischen Art seiner Internetverbindung und der Vermeidung zusätzlicher Soft- ware. Der eigene Internet-Browser reicht also immer aus, egal ob ein Büro-PC oder ein Rechner mit Mobilfunkverbindung verwendet wird. Drittens war bei der Entwicklung von Shibboleth der Datenschutz von sehr hoher Bedeutung. Mit Hilfe von Shibboleth bleibt Anbietern geschützter Ressourcen die individuelle Identität von Nutzern verborgen. Der Anbieter kann lediglich prüfen, ob hier ein berechtigter Nutzer auf das Angebot zugreifen möchte. Dieses Verfahren ist seit vielen Jahren u. a. bewährte Praxis für Einzelnutzer von Nationallizenzen.

Die vom KfL eingesetzte Proxy-Technik kann aber auch Berechtigungen anhand von IP-Adressen prüfen. Bei diesem Verfahren wird ermittelt, welche Adresse der zugreifende Rechner besitzt. Ist diese Adresse in einer Positivliste des Proxy verzeichnet, wird der Proxy den Zugriffswunsch an das eigentliche Zielsystem vermitteln.

\subsubsection{Systemarchitektur ${ }^{26}$}

Das System besteht aus sieben verschiedenen Komponenten:

- Proxies: Sie dienen hier der Lastverteilung und des Schutzes der eigentlichen KfL-Infrastruktur. Sämtliche Kommunikation mit der KfL-Infrastruktur wird über diese Proxies abgewickelt, welche nicht mit den oben beschriebenen identisch sind.

- Autorisierung/Authentifizierung: Die Basis bildet auch hier Shibboleth. Hierdurch wird das KfL selbst, mit allen oben geschilderten Vorteilen shibbolethfähig. In der Terminologie von Shibboleth übernimmt das KfL die Aufgabe eines Service Providers (SP).

- Identitätsmanagement (IdM): Es umfasst die Verwaltung der einzelnen FID-Nutzer, aber auch der Mitarbeiter eines FID und der Serviceteams des KfL. Mit Hilfe der Benutzerverwaltung werden Personen unterschiedliche Rollen zugeordnet, die mit unterschiedlichen Rechten versehen sind.

- Identity Provider (IdP): So wird im Kontext von Shibboleth die Technik bezeichnet, mit der ein SP kommunizieren muss, um Nutzer zu authentifizieren. Über den IdP wird definiert, welche Eigenschaften ein bestimmter Nutzer besitzt. Liegen die notwendigen Eigenschaften, die zur Nutzung einer elektronischen

26 Für die KfL-interne Dokumentation und Organisation besitzt das KfL auch ein eigenes Wiki und ein eigenes Ticketsystem. An dieser Stelle wird, weil selbstverständlich, darauf ebenso wenig eingegangen, wie auf die Monitoring-Werkzeuge oder die eingesetzte ServerInfrastruktur. 
Ressource berechtigen, vor, so kann der SP dem Nutzer den Zugriff gestatten.

- Electronic Resource Management System (ERMS): Das hier eingesetzte ERMS ist ein sowohl auf die Erfordernisse eines FID als auch auf die Anforderungen des KfL optimiertes Spezialsystem. Es unterstützt beide Seiten in allen Phasen der Lizenzverwaltung, beginnend bei der Akquise bis hin zur Präsentation. An diese Komponente werden besonders hohe Anforderungen gestellt. So muss sie nicht nur während Wartungsarbeiten, sondern auch nach einem Desaster eindeutig und korrekt den aktuellen Lizenzstatus abbilden. Daher ist diese Komponente dreifach vorhanden. Zwei Instanzen sorgen für einen unterbrechungsfreien Betrieb während Wartungsarbeiten und dienen ansonsten der internen Lastverteilung. Die dritte kommt dem flüssigen Arbeiten mit dem ERMS zugute. Ziehen einzelne Arbeitsschritte komplexe, kaskadierte Änderungsprozesse nach sich, können diese asynchron im Hintergrund abgearbeitet werden. Längere Wartepausen werden für Anwender so vermieden. Natürlich müssen alle drei Instanzen zueinander inhaltlich synchron gehalten werden.

- Objektdatenbank: Sie bildet quasi das Gedächtnis des KfL für Lizenzmodelle, Produkte und dergleichen.

- Relationales Datenbank Management System (RDBS): Die letzte Komponente ist ein RDBS.Das RDBS bildet die Grundlage für Export- bzw. ReportingSchnittstellen des KfL-Systems. Über die KfL-Schnittstellen lassen sich prinzipiell sämtliche vom KfL verwalteten Informationen rechtegesteuert abfragen. Auch diese Komponente erfüllt mehrere Aufgaben: Zum einen wird das Hauptsystem selbst bei komplexen und rechenintensiven Anfragen in der Performance nicht beeinträchtigt. Zum anderen müssen sämtliche Schnittstellen, die mit der „Außenwelt“ kommunizieren, besonders abgesichert werden. Das RDBS schützt das KfL-System vor Überlastungen durch möglicherweise unsachgemäße Verwendung der Schnittstelle und kapselt die externe Kommunikation vom Hauptsystem ab.

\subsection{Registrierung und Authentifizierung ${ }^{27}$}

Für die FID-Ressourcen nach dem Standardlizenzmodell FIDNutzerkreis erfolgt die Registrierung der Nutzer online

27 Vgl. Huber, Maria: Technische Infrastruktur des KfL zur Bereitstellung von FID-Lizenzen. Bedarfsgerechte Planung. Bibliothekartag über die Bereitstellungsplattform des KfL. Das KfL unterstützt den FID durch die Bereitstellung der erforderlichen technischen Infrastruktur, der Workflows, eines Electronic Resources Management Systems (ERMS) mit eigener Oberfläche für jeden FID und durch Beratungsleistungen.

Jedem FID wird eine FID-spezifische Sicht auf das KfLERMS angeboten, über den er seine Lizenzmodelle und seinen individuellen fachspezifischen Nutzerkreis verwalten kann. Auch die Endnutzer (Institutionen und Einzelnutzer) haben über diese FID-Oberfläche Zugang zur Anmeldung, zu ihrem Nutzerprofil und zu den Lizenzinformationen des FID. In die eigene Webpräsenz des FID, sofern vorhanden, ist diese FID-spezifische Anmeldeseite vom FID passend einzubinden, um den Benutzern einen direkten Zugang zur Registrierung für die FID-Lizenzen und zu ihrem FID-Nutzerprofil zu ermöglichen.

Registrieren können sich Institutionen sowie Einzelnutzer, die diesen Institutionen zuzuordnen sind. Die Registrierung von Einzelnutzern mit definierter Institutionszugehörigkeit wird immer angeboten und voraussichtlich der Standard sein. Sofern die registrierte Institution über IP-Adressbereiche sauber zu greifen ist, ist für den Zugriff aus dem Institutionsnetz die Registrierung von zugehörigen Einzelnutzern nicht nötig.

Der FID leitet den Registrierungsprozess typischerweise durch eine Information an seinen Nutzerkreis hinsichtlich des Produktangebots und der Möglichkeit zur Registrierung ein. Zunächst registrieren sich die Institutionen über die FID-Oberfläche des ERMS, der FID prüft ihre Zugehörigkeit zu seinem definierten Nutzerkreis, vergibt die Berechtigung, und die Institutionen erhalten eine Information über ihre Freischaltung und ggf. ihre Zugangsdaten zum ERMS. Anschließend können sich die Einzelnutzer registrieren und wählen dabei aus den bereits erfolgreich registrierten Institutionen ihre Heimateinrichtung aus. Im Rahmen eines definierten Workflows erhalten sie zunächst automatisch eine institutionsspezifische Mitteilung über das von ihrer Institution gewünschte Procedere für den Nachweis der Zugehörigkeit. Nachdem der Einzelnutzer ggf. den erforderlichen Nachweis erbracht hat und nach Prüfung und Bestätigung durch den im ERMS hinterlegten Ansprechpartner der Institution, erhält der Einzelnutzer

2015. Vortragsfolien: https://opus4.kobv.de/opus4-bib-info/files/167 1/bibtag_huber_20150526_final_ohne-Animation.pdf; Huber, Maria: Information für Fachinformationsdienste zu Anmeldung, Nutzerverwaltung und Authentifizierung für FID-Lizenzen (Stand: 1.12.2014): ht tp://www.fid-lizenzen.de/ueber-fid-lizenzen/dateien/fid-info_regis trierung_und_authentifizierung_20141201-3. Dargestellt wird hier die aktuelle Standardlösung für die nutzerkreisspezifische Bereitstellung (Lizenz- und Bereitstellungsmodell FIDNutzerkreis). 
seine Zugangsdaten. Damit kann er sowohl auf sein Nutzerprofil im ERMS als auch auf alle Lizenzen des betreffenden FID zugreifen. Für Einzelnutzer ist die Freischaltung jeweils ein Jahr gültig. Die Registrierung mit Institutionszugehörigkeit muss jährlich erneut bestätigt werden.

Dieser Registrierungsworkflow ist flexibel und überlässt den einzelnen FID die Entscheidung, wie aktiv sie die Institutionen aus ihrem Nutzerkreis bei der Registrierung und Verwaltung der Einzelnutzer einbeziehen wollen. Je nach Bedarf kann ein FID diesen Part komplett selbst übernehmen oder aber an die bereits berechtigten Heimateinrichtungen delegieren. Über folgende Parameter kann ein FID den Grad der Einbeziehung der Institutionen regulieren:

- Die Registrierung einer Institution kann durch die Institution selbst oder aber alternativ durch den FID vorgenommen werden.

- Im Account der Institution kann eine E-Mail-Adresse der Institution oder aber alternativ des FID hinterlegt werden. So erfährt die Institution evtl. nichts von ihrem ERMS-Account.

- Im Account der Institution kann für die Belange der Einzelnutzerregistrierung die E-Mail-Adresse eines Ansprechpartners aus der Institution oder aber alternativ des FID hinterlegt werden. Das bestimmt, wer zukünftig über die Registrierung von Angehörigen dieser Institution informiert wird.

- Im Account der Institution wird eine Mitteilung hinterlegt, die Mitglieder bei ihrer Registrierung als Einzelnutzer darüber informiert, wie ihre Zugehörigkeit nachzuweisen ist. Der Text ist institutionsspezifisch frei wählbar und nach Bedarf können auch weitere Unterlagen, wie etwa ein Formular zur schriftlichen Bestätigung der Zugehörigkeit eines Einzelnutzer durch seine Heimateinrichtung, eingebunden werden.

Die bisherigen Erfahrungen zeigen, dass die FID diese Parameter flexibel nutzen - das Spektrum reicht bereits vom FID, der die Einzelnutzerverwaltung an die Institution (en) abgibt bis zu dem FID, der die Institutionen komplett aus dem Registrierungsprozess heraus hält.

Die Speicherung und Verwaltung der Nutzerdaten erfolgt in der Regel zentral beim KfL (IdM, s. o. unter 5.1.). Auf Wunsch der FID ist es jedoch auch möglich, die Nutzerdaten außerhalb des KfL und innerhalb der Infrastruktur des FID zu speichern und zu verwalten. Dafür ist erforderlich, auch die technische Infrastruktur für den Workflow zur Vergabe der Zugangsberechtigungen dort abzubilden. Die Nutzerdaten selbst sind für die FID über eine Standardschnittstelle (API) aus dem KfL-ERMS abrufbar.
Die Authentifizierung der Nutzer gegenüber den Verlagen erfolgt zentral durch das Authentifizierungsmodul des KfL. Für die Zugriffssteuerung werden shibbolethfähige Proxies eingesetzt, die folgende Authentifizierungsmethoden unterstützen:

- Authentifizierung für Einzelpersonen über Zugangskennung als Standard.

- Zusätzlich IP-gesteuerte Authentifizierung für Institutionen, die eindeutig über IP-Adressen abgrenzbar sind.

Darüber hinaus ist geplant, eine Single-Sign-on-Authentifizierung gegenüber shibbolethfähigen Heimateinrichtungen zu ermöglichen.

Jeder FID kann im ERMS zunächst einen einzigen Nutzerkreis definieren, der produktunabhängig für alle Lizenzen dieses FID gleich ist. Die technische Abbildung lizenzspezifischer heterogener Nutzerkreise und Beteiligungsmodelle wie FIDOpt-In und FIDSelected ist in Planung, derzeit im ERMS aber noch nicht realisiert.

Die Bereitstellungsplattform des KfL ermöglicht angemeldeten Einzelnutzern, die interdisziplinär forschen, den komfortablen Wechsel zwischen den Angeboten ihrer FID.

\subsection{Bereitstellungsmodelle und Kommunikationsstrategien}

Die Bereitstellungsmodelle für die FID-Lizenzen stehen in enger Relation zu den betreffenden Nutzerkreis- und Lizenzmodellen. Es haben sich hier bislang analog zu den Lizenzmodellen die folgenden drei Bereitstellungsmodelle herauskristallisiert, die auch unterschiedliche Kommunikationsstrategien zur Information über die Lizenzen erforderlich machen:

- FIDNutzerkreis: FID-Lizenz für einen definierten Nutzerkreis, d.h. über eine einzelne Einrichtung hinausgehend.

Die Bereitstellung der Lizenz und Registrierung institutionsbezogener Einzelnutzer erfolgt über die Plattform des KfL, vermittelt durch die Webpräsenzen der FID.

Die Information und Kommunikation der Bereitstellung sowie der Aufruf zur Registrierung erfolgt durch die FID, adressiert an den jeweiligen Nutzerkreis.

Die dafür eingesetzten Kommunikationsstrategien sind FID-spezifisch, die Adressaten werden FID-spezifisch gewählt.

- FIDCampus: FID-Lizenz zur campusweiten Freischaltung für alle Institutionen eines definierten Nutzerkreises. 
Die campusweite Bereitstellung erfolgt IP-gesteuert, derzeit ist hier kein Anmeldeverfahren erforderlich. Das KfL unterstützt den Prozess der IP-Freischaltung. Die Information und Kommunikation der Bereitstellung erfolgt durch die FID, adressiert an den jeweiligen Nutzerkreis.

Die dafür eingesetzten Kommunikationsstrategien sind FID-spezifisch, Adressaten sind die Bibliotheken der im Nutzerkreis gelisteten Institutionen. Das KfL unterstützt den FID mit den entsprechenden Kontaktinformationen.

- FIDNationallizenz: Klassische Nationallizenz ohne (fachliche) Einschränkung hinsichtlich der Nutzergruppe (Nationallizenz).

Die Bereitstellung der Lizenz und die Registrierung von Institutionen erfolgt über www.nationallizenzen. de, nach dem bekannten Verfahren für die DFG-geförderten Nationallizenzen. Die Freischaltung wird IP-gesteuert vorgenommen.

Die Information und Kommunikation der Bereitstellung erfolgt durch die FID sowie durch das KfL, adressiert an den jeweiligen Nutzerkreis (FID) und den Mailverteiler der Nationallizenzen (KfL).

Das Bereitstellungsmodell FIDNutzerkreis stellt die Standardlösung dar, dem das Gros der FID-Lizenzen zuzuordnen ist.

Bei den Lizenzmodellen FIDCampus und FIDNationallizenz werden die Bibliotheken der zugangsberechtigten Einrichtungen vom FID bzw. vom KfL über die Bereitstellung der Lizenz in Kenntnis gesetzt. Hier sind die Bibliotheken auch in den Bereitstellungsprozess involviert, der jeweils nach dem für Campus- und Nationallizenzen etablierten bekannten Verfahren erfolgt. Ein Zugriff ist so z.B. erst nach Anerkennung der Nutzungsbedingungen möglich.

In den Verfahren um die Bereitstellung von FID-Lizenzen nach dem Modell FIDNutzerkreis sind die Bibliotheken meist nicht beteiligt. Hier laufen die Prozesse im direkten Austausch zwischen FID und den Wissenschaftlern des Nutzerkreises, ggf. administrativ unterstützt durch das KfL. Das KfL übernimmt auf Wunsch der FID auch die Verwaltung und Erschließung der Lizenzen. Eine indirekte allgemeine Information über die Bereitstellung der Lizenzen erfolgt über die Produktliste auf der Website des KfL ${ }^{28}$ sowie über den Nachweis der FID-Lizenzen in den Katalogen und einschlägigen Nachweissystemen.

28 Vgl. http://www.fid-lizenzen.de/angebote.

\subsection{Erschließung von FID-Lizenzen in den Nachweissystemen}

Das Kompetenzzentrum für Lizenzierung (KfL) unterstützt auf Wunsch die FID beim Einbringen der FID-Produkte in die zentralen Nachweissysteme ZDB, EZB, DBIS und die Verbundkataloge. Dabei gibt der FID vor, welche Nachweise in welchen Nachweissystemen vorgenommen werden sollen. Die Nachweissituation für die FID-Lizenzen ist derzeit noch nicht abschließend gelöst, es besteht hier sowohl seitens der Nachweissysteme als auch seitens der FID noch Klärungsbedarf. ${ }^{29}$

\section{Perspektiven}

Mit dem Kompetenzzentrum für die Lizenzierung elektronischer Ressourcen im FID-Kontext ist im Rahmen der Beschaffungsstrukturen für elektronische Ressourcen erstmals eine zentrale Instanz für die Lizenzierung elektronischer Medien in Deutschland etabliert worden. Das KfL hat seine Verhandlungstätigkeit sofort zu Projektbeginn aufgenommen, die Betriebsabläufe sind inzwischen voll entwickelt und in der Praxis belastbar erprobt, allen definierten Anforderungen wird voll entsprochen. Das KfL wird im Rahmen der verbleibenden Projektlaufzeit die Modellentwicklung für die FID-Lizenzen, den weiteren Ausbau des KfL-ERMS sowie die Verzeichnung von FID-Lizenzen in den Nachweissystemen weiter vorantreiben und seine Serviceangebote im Dialog mit den FID weiter entwickeln.

Parallel dazu hat sich das KfL im Rahmen der etablierten Beschaffungsstrukturen für die überregionale Lizenzierung von elektronischen Medien in Deutschland eingefügt und mit zentralen Instanzen vernetzt (GASCO, Allianz-AG Lizenzen, DBV-Erwerbungskommission). Über seine Website stellt das KfL eine fachübergreifende, zentrale Liste der FID-Lizenzen bereit, die von den durch das KfL betreuten FID angeboten werden. ${ }^{30}$ Das KfL möchte damit Konsortien und Bibliotheken eine Orientierungshilfe geben. Weitere Entwicklungen und parallele Handlungsfelder auf nationaler Ebene werden stets im Blick behalten.

Unter Entwicklung der eigenen Möglichkeiten und in Kooperation mit weiteren relevanten Playern im Kontext

29 Zum aktuellen Diskussionsstand vgl. Husaini, Masen; Stanek, Ursula: Information für Fachinformationsdienste zum Nachweis von FID-Lizenzen (Stand: 1.9.2015): http://www.fid-lizenzen.de/ueber-fi d-lizenzen/dateien/FIDInfo_FIDLizenzeninNachweissystemen.pdf. 30 Vgl. http://www.fid-lizenzen.de/angebote. 
der überregionalen Lizenzierung elektronischer Medien in Deutschland bleibt es das Ziel des KfL, einen bedarfsorientierten individuellen Service zur Unterstützung der FID bei der Wahrnehmung ihrer Aufgaben für die Fachcommunities zu leisten.

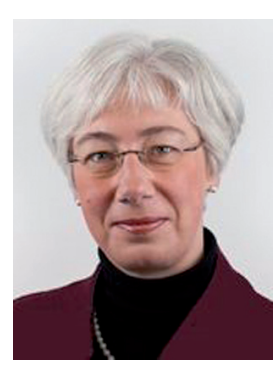

\section{Kristine Hillenkötter}

Niedersächsische Staats- und Universitätsbibliothek Göttingen

Platz der Göttinger Sieben 1

D-37073 Göttingen

hillenkoetter@sub.uni-goettingen.de

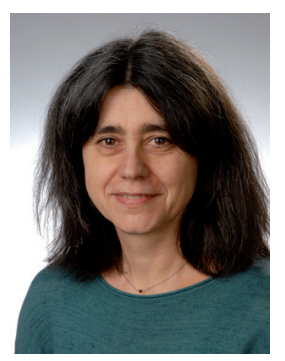

\section{Maria Huber}

Niedersächsische Staats- und Universitätsbibliothek Göttingen

Platz der Göttinger Sieben 1

D-37073 Göttingen

maria.huber@sub.uni-goettingen.de

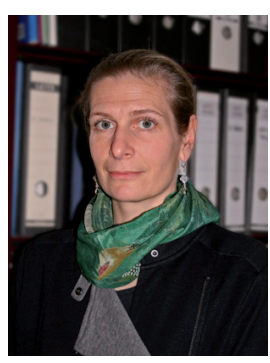

Dr. Ursula Stanek

Staatsbibliothek zu Berlin - Preußischer Kulturbesitz

Potsdamer Str. 33

D-10785 Berlin

ursula.stanek@sbb.spk-berlin.de

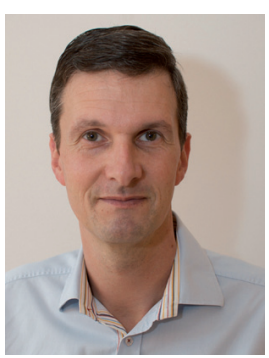

\section{Gerald Steilen}

Verbundzentrale des GBV (VZG)

Platz der Göttinger Sieben 1

D-37073 Göttingen

steilen@gbv.de 